\title{
Tracing Uncontrolled Asthma in Family Practice Using a Mailed Asthma Control Questionnaire
}

\author{
Lotte van den Nieuwenhof, $M D^{1}$ \\ Tjard Schermer, $P b D^{1}$ \\ Marianne Heins, MSc ${ }^{1}$ \\ Joke Grootens, $B c^{1}$ \\ Petra Eysink, $\mathrm{PbD} \mathrm{D}^{2}$ \\ Ben Bottema, MD, $P b D^{2}$ \\ Chris van Weel, $M D, P b D^{1}$ \\ Patrick Bindels, $M D, P b D^{2}$ \\ 'Department of Family Practice, Radboud \\ University Nijmegen Medical Centre, \\ Nijmegen, the Netherlands \\ ${ }^{2}$ Department of Family Practice, Academic \\ Medical Centre-University of Amsterdam, \\ Division of Clinical Methods and Public \\ Health, Amsterdam, the Netherlands
}

Conflicts of interest: Lotte van den Nienwenhof bas received unrestricted educational and research grants for bis department from AstraZeneca, Boebringer Ingelheim, GlaxoSmitbKline, and Novartis; Marianne Heins, Joke Grootens, Petra Eysink, and Ben Bottema declare no conflicts of interest. Tjard Schermer has received unrestricted research grants from GlaxoSmithKline, Boebringer Ingelbeim, and AstraZeneca, Chris van Weel has a nonpersonal interest of less than $\$ 10,000$ in AstraZeneca, Bayer, and NovNordisk, and a nonpersonal interest of more than $\$ 10,000$ in Boebringer Ingelheim, GlaxoSmithKline, and Novartis; and Patrick Bindels bas received unrestricted research grants from GlaxoSmithKline.

\section{CORRESPONDING AUTHOR}

Lotte van den Nieuwenhof, MD

Department of Family Practice (117-HAG)

Radboud University

Nijmegen Medical Centre

PO Box 9101

$6500 \mathrm{HB}$ Nijmegen, the Netherlands

L.vandennieuwenhof@hag.umcn.nl

\begin{abstract}
PURPOSE A substantial proportion of adult patients with asthma have inadequately controlled symptoms despite the availability of effective treatment. The Asthma Control Questionnaire (ACQ) can be used to discriminate between asthma patients with well- and suboptimally controlled asthma symptoms. The objective of this study was to investigate whether a postal mailing of the ACQ can be used to identify asthma patients with suboptimal symptom control in family practice.
\end{abstract}

METHODS In this observational study, we sent 434 asthma patients from 6 Dutch family practices an ACQ by mail to measure control of their asthma symptoms. Both respondents and nonrespondents were characterized by information gathered from their medical records. Patients with an ACQ sum score (total score) of greater than 3 were considered to have suboptimally controlled asthma symptoms.

RESULTS The response rate was $77 \%$. Respondents were more likely than nonrespondents to be female and to use asthma medication. The mean ACQ sum score of the respondents was 5.2. Of this group, 53.4\% (95\% confidence interval, 48.0\%-58.8\%) had suboptimally controlled asthma symptoms. Of the 168 respondents who had not visited their family physician in the 2 years before the study, $42.9 \%$ (95\% confidence interval, 35.4\%-50.4\%) had inadequate asthma symptom control.

CONCLUSIONS Our results show that a postal mailing of the ACQ is an effective approach for tracing asthma patients who need medical attention. It also traces patients who would otherwise not have consulted their family physician. The ACQ seems to be a useful starting point for health care professionals in family practice to improve the level of asthma symptom control in their patient population.

Ann Fam Med 2008;6(suppl 1):s16-s22. DOI: 10.1370/afm.776.

\section{INTRODUCTION}

$\mathrm{P}$ opulation surveys have shown that despite the availability of highly effective pharmacotherapy, the majority (up to $70 \%-95 \%$ ) of all asthma patients in western Europe and the Asia-Pacific region have signs of poor asthma control. ${ }^{1-3}$ Known causes of this suboptimal level of asthma control are poor adherence to periodic management visits to health care professionals $\mathrm{s}^{4-6}$ or insufficient compliance with prescribed asthma medication, especially inhaled corticosteroids. ${ }^{5}$ In addition, patients with asthma - and their physicians - tend to overestimate their level of asthma control. ${ }^{78}$ These factors emphasize the need to improve the identification of poor asthma control and subsequent treatment. Because the majority of patients with asthma are treated by family physicians, family practice would be the most appropriate setting to study the tracing of patients with poor asthma control.

Bronchial inflammation is a marker of asthma control that can be assessed by induced sputum eosinophilia ${ }^{9}$ or bronchial hyperrespon- 
siveness. ${ }^{10}$ The requirement of laboratory facilities, the time-consuming nature of these tests, and their unfriendliness to patients severely hamper their routine use in primary care, however. An increase of asthma symptoms has been shown to indicate a loss of asthma control, ${ }^{11,12}$ making measurement of changes in symptoms an attractive alternative to the above tests.

In a previous study, ${ }_{1}^{13}$ we found that the Asthma Control Questionnaire $(\mathrm{ACQ})^{14-16}$ can be used to distinguish asthma patients with good symptom control from those with suboptimal symptom control. The aim of the current study was to investigate whether a mailing of the ACQ can be used to trace asthma patients with suboptimal symptom control in the family practice population.

\section{METHODS}

\section{Design and Patient Recruitment}

This was a multicenter observational study conducted in 6 family practices from the Amsterdam $(n=3)$ and Nijmegen $(n=3)$ regions in the Netherlands. In the Netherlands, patients are registered with a family physician, who largely coordinates access to health care services. The study was approved by the medical ethics review board of the Radboud University Nijmegen Medical Centre.

We selected patients with asthma from the practices' computerized medical records using 2 inclusion criteria: (1) an age of 18 to 45 years and (2) a diagnostic label of asthma and/or, during the 2 years preceding the study, at least 1 prescription for an inhaled corticosteroid and/ or at least 2 prescriptions for an inhaled bronchodilator. The family physicians received a list of all selected patients in their practice and were asked to exclude any who had a diagnosis of chronic obstructive pulmonary disease or who were unable to communicate in Dutch.

\section{Measures}

For all patients, we extracted the following information from their family practice medical records: (1) sex, (2) age, and (3) in the 2 years preceding the study, number of visits to the family physician for asthma-related complaints, asthma exacerbations, prescriptions for asthma medication ( $\beta_{2}$ agonists, anticholinergics, inhaled and oral corticosteroids, cromoglycates, leukotriene antagonists), referrals to chest physicians, asthma-related emergency department visits, and hospital admissions.

In an initial mailing, patients were sent the ACQ from their family physician with a cover letter and a prepaid return envelope. In a reminder mailing, sent to patients who had not responded 4 weeks after the initial mailing, patients received a reminder letter along with another ACQ and another prepaid return envelope.

The $\mathrm{ACQ}^{14}$ contains a set of questions that allow patients with asthma to rate the severity of their respiratory symptoms (Table 1 ). Patients are asked to recall their respiratory health status during the previous week and respond to each question on a 7-point scale ranging from 0 (well controlled) to 6 (extremely poorly controlled). The ACQ has been validated and has been

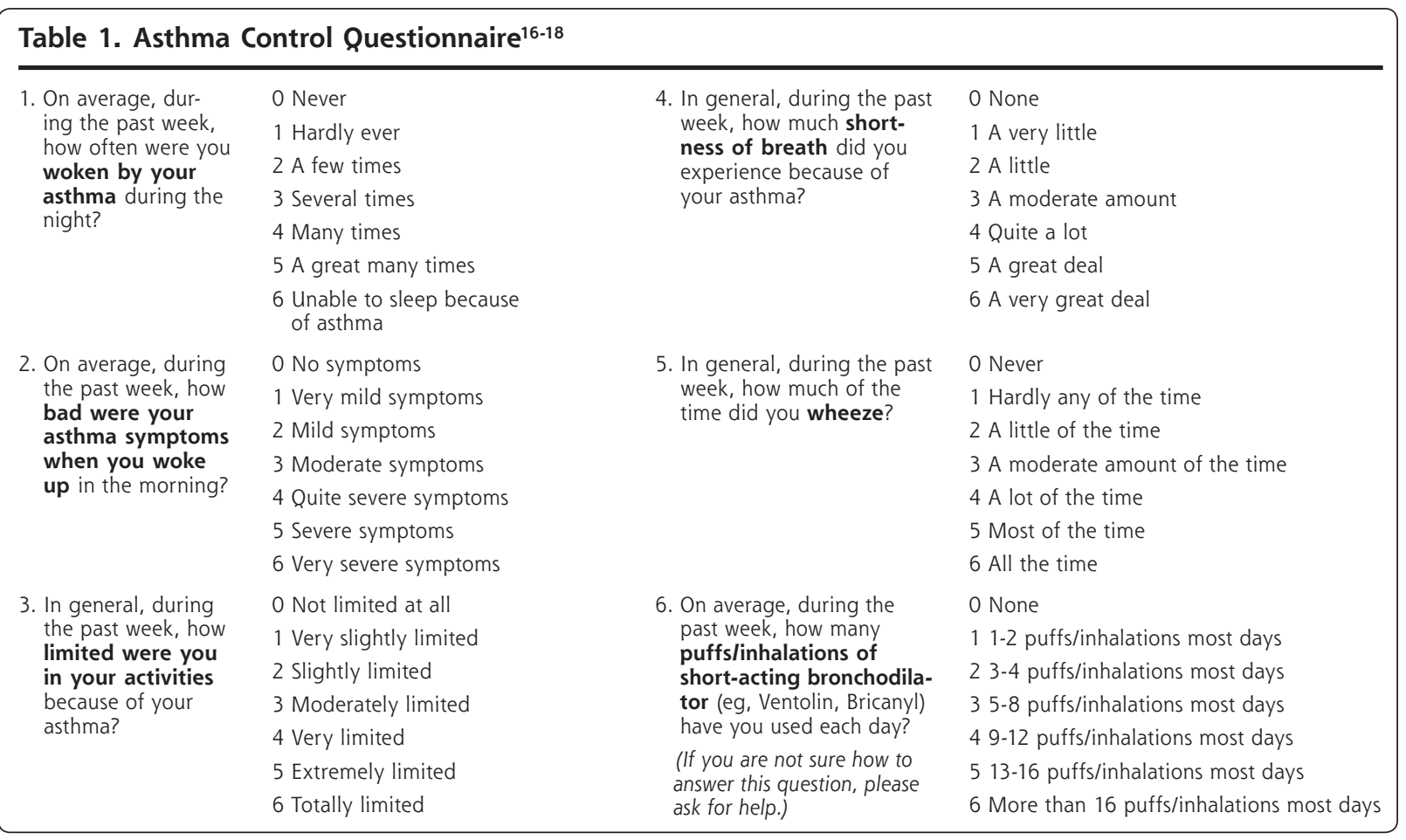


used to measure the effects of asthma treatment in clinical studies. ${ }^{14-17}$ Initially, the questionnaire included a question on pulmonary function, but omission of that question did not influence the validity of the ACQ. ${ }^{16,18}$ The questionnaire was therefore modified to the 6 question $A C Q$ used in this study. ${ }^{16-18}$

\section{Analysis}

We used the sum score (total score) of the ACQ's 6 questions (range, 0-36 points) - instead of the mean score, which is generally used-because this is easier to calculate in daily practice. As long as patients answer all questions, the interpretation is the same. The higher the sum score, the poorer patients' asthma control.

In a previously reported pilot study, ${ }_{1}^{13}$ we established an ACQ cutoff value for identifying patients with asthma who have suboptimal control of their symptoms. A sum score of 3 points or less best indicated good asthma symptom control, having a sensitivity of $84 \%$ and a specificity of $76 \%$.

A primary respondent was defined as a patient who returned the ACQ after the first mailing, and a secondary respondent was defined as a patient who returned the ACQ after the reminder mailing. Nonrespondents were patients who did not return the questionnaire after these 2 consecutive attempts. Patients who had visited their family physician for asthma-related complaints during the 2-year period before the mailing were defined as visitors, and those who had not were defined as nonvisitors.

We performed data analysis with SPSS version 12.0 for Microsoft Windows (SPSS Inc, Chicago, Illinois). Various study groups were compared with regard to the information extracted from their medical records and-apart from the nonrespondents - their ACQ sum scores. Specifically, we compared differences between respondents overall and nonrespondents, between primary and secondary respondents, and between visitors and nonvisitors using the $\chi^{2}$, Mann Whitney $U$, and 1-way ANOVA statistical tests. A 95\% confidence interval $(\mathrm{CI})$ was calculated for the most relevant proportions.

\section{RESULTS}

Mailing Response and Study Population

We identified and sent the ACQ to 434 patients with asthma from the 6 family practices who met the inclusion and exclusion criteria. Patient flow in the study is shown in Figure 1. A total of 333 patients or $76.7 \%$
(95\% CI, $72.7 \%-80.7 \%)$ of all patients who were sent the ACQ returned it.

Characteristics of the respondents overall and nonrespondents are shown in Table 2 . Compared with nonrespondents, respondents were more likely to be female $(62.5 \%$ vs $45.5 \%)$, were slightly older (34.6 vs 32.6 years), and were more likely to be using asthma medication in general $(79.6 \%$ vs $61.4 \%)$ and inhaled corticosteroids in particular $(28.8 \%$ vs $16.8 \%)$. We found no other statistically significant differences between these groups.

Characteristics of the primary respondents and secondary respondents are shown in Table 3. Compared with secondary respondents, primary respondents consulted their family physician more frequently $(1.3$ vs 0.8 times in the preceding 2 years) and were more likely to be using any asthma medication (83.2\% vs $69.7 \%$ ). These groups were otherwise statistically indistinguishable.

\section{Symptom Control in Respondents and Nonrespondents}

In the 2 years preceding the mailing, in the nonrespondent group, 1 patient had been admitted to the hospital
Figure 1. Patient flow.

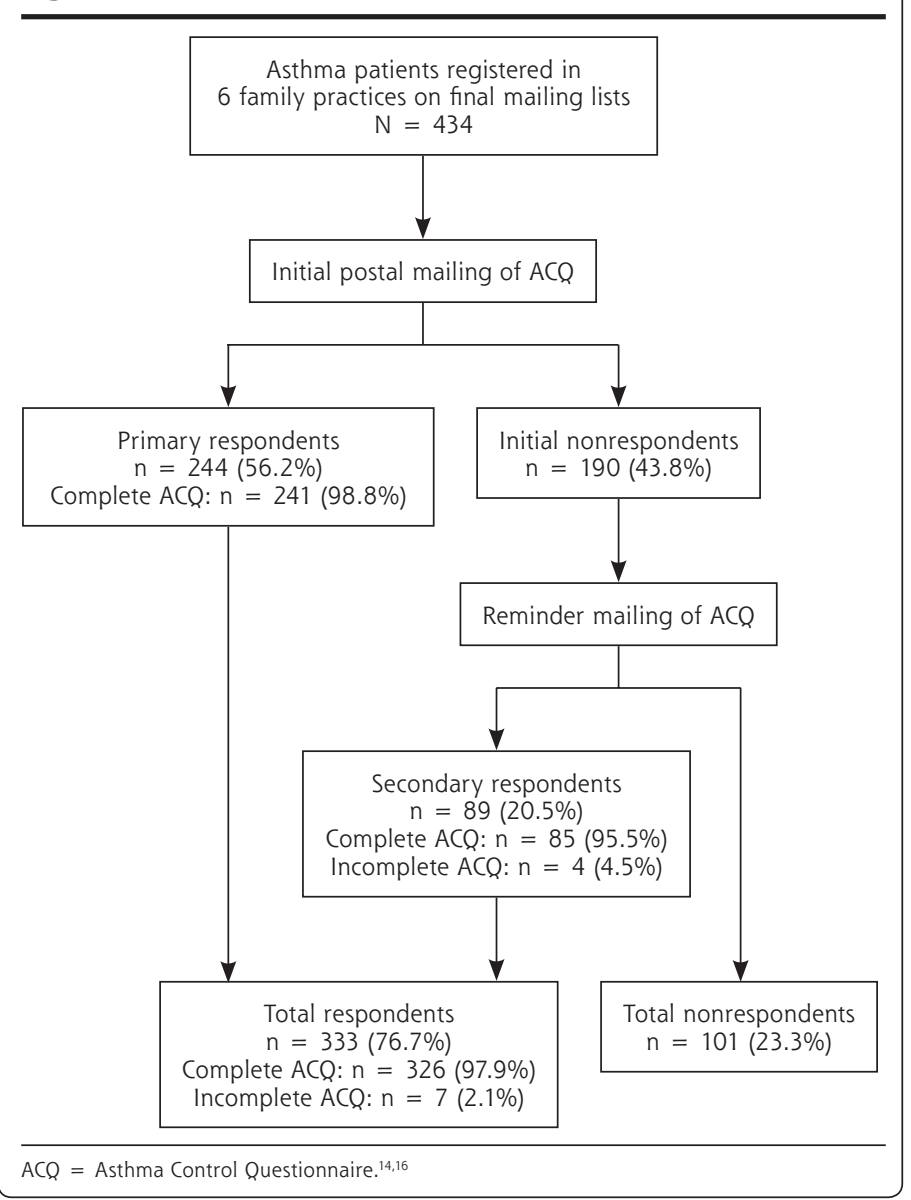


and 1 patient had visited an emergency department because of an acute asthma attack. None of the patients in the respondent group had experienced either of these events.

The mean ACQ sum score of the respondents was 5.2 points (Table 2 ). Secondary respondents had a lower ACQ sum score than primary respondents (4.8 vs 5.3 points), although the difference was not significant (Table 3). The distribution of the ACQ sum scores among all respondents is depicted in Figure 2. When primary and secondary respondents with a complete ACQ were combined $(\mathrm{n}=326)$, fully $53.4 \%$ (95\% CI, 48.0\%-58.8\%) of respondents had symptoms of suboptimally controlled asthma (ie, an ACQ sum score $>3$ points).

\section{Symptom Control in Visitors and Nonvisitors}

A total of 207 of the patients who received the mailed ACQ from their family physician, or $47.7 \%$ (95\% CI, $43.0 \%-52.4 \%)$, met the definition of visitors because they had at least 1 documented asthma-related family physician visit during the preceding 2 years. Of the 174 respondents with suboptimally controlled symptoms, $58.6 \%$ had at least 1 visit. According to their medical records, visitors had had an average of 2.4 (SD, 2.0) asthma-related consultations.

Patients completing the ACQ were nearly equally split by visitation status: $158(48.4 \%)$ were visitors and 168 (51.6\%) were nonvisitors. Compared with the visitors, the nonvisitors had better asthma symptom control: the mean ACQ sum score was 3.9 points $(\mathrm{SD}, 4.5)$ for nonvisitors and 6.5 points $(\mathrm{SD}, 5.6)$ for visitors $(P<.001)$. Still, $42.9 \%$ (95\% CI, 35.4\%-50.4\%) of the 168 nonvisitors who returned their ACQ had suboptimal symptom control (ie, an ACQ sum score $>3$ points), although this was less than the $64.6 \%$ value among visitors.

\section{DISCUSSION}

A main goal in the management of asthma is to achieve optimal control of respiratory symptoms, ${ }^{20}$ but recent surveys show that there is considerable room for improvement with regard to the level of asthma control in the general population. ${ }^{1-3}$ As best we could determine, no other studies have attempted to identify patients with poor asthma symptom control in a primary care setting with the aid of a simple method. The main objective of our study, therefore, was to evaluate whether a mailing of the ACQ can be used to identify patients with suboptimal symptom control among all patients registered in a family practice who have asthma.

With the ACQ mailing, we were able to reach $77 \%$ of all the asthma patients registered in the participating family practices, including a rather large group of patients who had not had a single contact for respiratory complaints with their family physician in the past 2 years. When using our previously determined cutoff value of an ACQ sum score of greater than 3 , we found that about 1 out of every 2 asthma patients (53\%) in these family practices were classified as having suboptimally controlled asthma symptoms. A somewhat higher prevalence of suboptimal asthma control $(60 \%)$ has been reported earlier. ${ }^{21}$

Other researchers have found that the cutoff value 
of 3 has as sensitivity of $90 \%$ and a specificity of $58 \%{ }^{17}$; however, the ACQ cutoff value that is most appropriate depends on the health care professional's goals. When resources are limited, the goal might be to identify only patients with very poorly controlled asthma symptoms, and a higher ACQ cutoff might be more appropriate. ${ }^{13}$

An interesting observation was that a substantial proportion of the visitors (65\%) still had suboptimal symptom control. In fact, this proportion was even greater than that among the nonvisitors (43\%). From these findings, we conclude that the largest number of patients with suboptimal control of their asthma was found among those who had visited their family physician recently for asthma. This association indicates that regular consultation of the family physician does not always indicate well-controlled asthma. It has previously been shown that patients as well as their physicians do not always adequately recognize suboptimal asthma control, ${ }^{7,8}$ which possibly explains part of this pattern. On the other hand, because asthma is usually treated as an episodic disorder, asthma patients who visit their family physician probably have more asthma-related problems. This study provides empiric data that this is the case, but also that the situation is more complex than that, given that poor control was also common among nonvisitors.

Although mailing visitors an ACQ is a feasible way to identify suboptimal asthma control, as this study has demonstrated, we question the relevance of this approach for this group. Identification of poorly controlled symptoms is only the initial step, and it should lead to better management. For these visitors, a more efficient approach would in all probability be to include the ACQ in their regular follow-up visits. Judging from our findings, that approach would identify the largest number of patients with poorly controlled asthma in the family practice population.

Mailing the ACQ does make sense for the population of patients who have asthma but do not make regular asthma visits to their family physician. Our findings confirm that this is a substantial proportion of the asthma population. An interesting area for further study would be the follow-up on this finding. A logical next step for the nonvisitors would be to invite those with high ACQ sum scores (eg, $>3$ points) to visit their family physician or practice nurse. This visit could be used to identify yet unidentified triggers for the poor asthma symptom control, discuss the patients' personal preferences, and evaluate their current asthma management, 
which is likely to offer substantial room for improvement. ${ }^{22,23}$ As $57 \%$ of the nonvisitors have well-controlled symptoms and do not need an extra visit, the ACQ can therefore be used to efficiently identify which nonvisitors need extra attention. We expect, however, that fewer patients with suboptimally controlled asthma will be found among the nonvisitors than among the visitors. As the nonvisitors did not visit their family physician on their own initiative, one might ask whether they would respond to an invitation to an asthma review; however, their completion of the ACQ might indicate that they are not unwilling but only unaware of their poor asthma control. This would be an interesting area for further study.

In this study, we evaluated a postal mailing with a single reminder mailing after 4 weeks in the case of initial nonresponse. We considered this approach to be feasible for any family practice. More than onehalf of all approached patients responded to the initial questionnaire mailing, and the response rate further increased to three-quarters of all approached patients after a reminder was sent.

Secondary respondents did not have more asthma problems than primary respondents. One might have expected that patients who were reluctant to respond to a health questionnaire would also be reluctant to follow treatment. But judging from the rates of poor asthma control, there are no indications that secondary respondents fared worse in their asthma (self-) management. It would be interesting to know how this finding relates to that among patients who did not respond at all to the ACQ. Although we regard our response rate to be satisfactory, no information on the current level of symptom control could be gathered in 1 of every 4 asthma patients. Relative to respondents, nonrespondents were more likely to be male and were less likely to use inhaled corticosteroids. Other investigators have also reported that men are less likely than women to respond to postal mailings. ${ }^{24,25}$ It has been suggested that when using symptom-orientated questionnaires, individuals with health problems may respond more often than those without. ${ }^{26} \mathrm{On}$ the other hand, a report from an epidemiologic study on respiratory health found that nonrespondents experienced more respiratory symptoms than respondents did. ${ }^{25}$ It is therefore possible that some of the asthma patients in our study who did not return questionnaires experienced more symptoms than their responding counterparts, and their lack of response may have concealed a poor level of asthma control among the nonrespondents. The information obtained from the medical records did not, however, point to a higher rate among the nonrespondents of respiratory-related family physician consultations, hospitalizations, or emergency department visits for asthma, or respiratory medication use.

When selecting patients with asthma for inclusion in this study, we could use only the information that was routinely recorded in the family physicians' computerized patient journal system. We could therefore not verify if every patient on the mailing list actually met the current national ${ }^{27}$ or international ${ }^{20}$ diagnostic criteria for asthma. Because patient records may not always be up to date with regard to the labels attached, our reliance on records may have led to some misclassification of patients selected for the mailing.

In conclusion, we found that the majority of adult asthma patients registered in Dutch family practices responded to a postal mailing with a short questionnaire regarding asthma symptom control-in our case, the ACQ. This mailing reached asthma patients who would otherwise not have come to the attention of the family physician. In this group of patients, there certainly seemed to be room for improvement in management given the apparently high prevalence of suboptimal control of their asthma symptoms. Our findings suggest that a postal mailing of the ACQ may be an appropriate starting point for identifying patients in a family practice who have suboptimal control of asthma symptoms.

To read or post commentaries in response to this article, see it online at http://www.annfammed.org/cgi/content/full/6/suppl_1/s16.

Submitted April 18, 2007; submitted, revised, July 13, 2007; accepted August 1, 2007.

Key words: Asthma; disease management; ACQ; questionnaires; mail; family practice; primary care; practice-based research

Funding support: GlaxoSmithKline provided an unrestricted grant for this study.

Acknowledgments: The authors owe much gratitude to Mr Aad Wissink for his assistance with the study logistics and data collection, to all family practitioners and practices nurses from the involved practices, and to the patients who returned their copy of the ACQ.

\section{References}

1. Rabe KF, Vermeire PA, Soriano JB, Maier WC. Clinical management of asthma in 1999: the Asthma Insights and Reality in Europe (AIRE) study. Eur Resp J. 2000;16(5):802-807.

2. de Marco R, Bugiani M, Cazzoletti L, et al. The control of asthma in Italy. A multicentre descriptive study on young adults with doctor diagnosed current asthma. Allergy. 2003;58(3):221-228.

3. Lai CK, De Guia TS, Kim YY, et al. Asthma control in the Asia-Pacific region: the Asthma Insights and Reality in Asia-Pacific Study. J Allergy Clin Immunol. 2003;111(2):263-268.

4. Variations in the prevalence of respiratory symptoms, self-reported asthma attacks, and use of asthma medication in the European Community Respiratory Health Survey (ECRHS). Eur Respir J. 1996;9(4):687-695. 
5. Boulet LP. Perception of the role and potential side effects of inhaled corticosteroids among asthmatic patients. Chest. 1998; 113(3):587-592

6. Kips JC, Pauwels RA. Asthma control: where do we fail? Eur Resp J. 2000;16(5):797-798.

7. Boulet LP, Phillips R, O'Byrne P, Becker A. Evaluation of asthma control by physicians and patients: comparison with current guidelines. Can Respir J. 2002;9(6):417-423.

8. Juniper EF, Chauhan A, Neville E, et al. Clinicians tend to overestimate improvements in asthma control: an unexpected observation. Prim Care Respir J. 2004;(4):181-184.

9. Green RH, Brightling CE, McKenna S, et al. Asthma exacerbations and sputum eosinophil counts: a randomised controlled trial. Lancet. 2002;360(9347):1715-1721

10. Sont JK, Willems LN, Bel EH, van Krieken JH, Vandenbroucke JP, Sterk PJ. Clinical control and histopathologic outcome of asthma when using airway hyperresponsiveness as an additional guide to long-term treatment. The AMPUL Study Group. Am J Respir Crit Care Med. 1999;159(4 Pt 1):1043-1051.

11. Magnan A. Tools to assess (and achieve?) long-term asthma control. Respir Med. 2004;98(Suppl B):S16-S21.

12. Boulet LP, Boulet V, Milot J. How should we quantify asthma control? A proposal. Chest. 2002;122(6):2217-2223.

13. van den Nieuwenhof $L$, Schermer T, Eysink $P$, et al. Can the Asthma Control Questionnaire be used to differentiate between patients with controlled and uncontrolled asthma symptoms? A pilot study. Fam Pract. 2006;23(6):674-681

14. Juniper EF, O'Byrne PM, Guyatt GH, Ferrie PJ, King DR. Development and validation of a questionnaire to measure asthma control. Eur Respir J. 1999;14(4):902-907.

15. Juniper EF, O'Byrne PM, Ferrie PJ, King DR, Roberts JN. Measuring asthma control. Clinic questionnaire or daily diary? Am J Respir Crit Care Med. 2000;162(4 Pt 1):1330-1334.

16. Juniper EF, Svensson K, Mork AC, Stahl E. Measurement properties and interpretation of three shortened versions of the asthma control questionnaire. Respir Med. 2005;99(5):553-558.
17. Juniper EF, Bousquet J, Abetz L, Bateman ED. Identifying 'well-controlled' and 'not well-controlled' asthma using the Asthma Control Questionnaire. Respir Med. 2006;100(4):616-621.

18. Juniper EF, O'Byrne PM, Roberts JN. Measuring asthma control in group studies: do we need airway calibre and rescue beta2-agonist use? Respir Med. 2001;95(5):319-323.

19. National Institutes of Health. National Heart, Lung, and Blood Institute. Global Strategy for Asthma Management and Prevention (update 2004). Bethesda, MD: National Institutes of Health, National Heart, Lung, and Blood Institute; 2005. NIH publication 02-3659.

20. From the Global Strategy for Asthma Management and Prevention. Global Initiative for Asthma (GINA) 2006. http://www.ginasthma.org. Accessed July 10, 2007.

21. Wijnhoven HA, Kriegsman DM, Hesselink AE, Haan M, Stalman W. Disease control in general practice patients with asthma. Prim Care Respir J. 2004;13(2):89-98.

22. Bateman ED, Boushey HA, Bousquet J, et al. Can guideline-defined asthma control be achieved? The Gaining Optimal Asthma ControL study. Am J Respir Crit Care Med. 2004;170(3):836-844.

23. de Marco R, Cazzoletti L, Cerveri I, et al. Are the asthma guideline goals achieved in daily practice? A population-based study on treatment adequacy and the control of asthma. Int Arch Allergy Immunol. 2005;138(3):225-234

24. Kotaniemi JT, Hassi J, Kataja M, et al. Does non-responder bias have a significant effect on the results in a postal questionnaire study? Eur J Epidemiol. 2001;17(9):809-817.

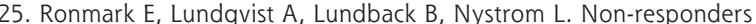
to a postal questionnaire on respiratory symptoms and diseases. Eur J Epidemiol. 1999;15(3):293-299.

26. Bakke P, Gulsvik A, Lilleng P, Overa O, Hanoa R, Eide GE. Postal survey on airborne occupational exposure and respiratory disorders in Norway: causes and consequences of non-response. J Epidemiol Community Health. 1990;44(4):316-320.

27. Geijer RMM, Thiadens HA, Smeele IJM, et al. NHG-Standaard COPD en Astma bij volwassenen: diagnostiek [NHG guideline on COPD and asthma in adults: diagnosis]. Huisarts Wet. 2001;44:107-117. 